ISSN: 0514-7336

DOI: https://doi.org/10.14201/zephyrus201678203209

\title{
NUEVA ÁRULA A ATAECINA EN OÑA (BURGOS)
}

\section{New altar to Ataecina in Oña (Burgos)}

\author{
Marta Fernández Corral \\ Dpto. de Estudios Clásicos. Facultad de Letras UPV-EHU. C/ Tomás y Valiente, s/n. 01006 Vitoria. Correo-e: marta. \\ fernandezc@ehu.eus
}

Recepción: 24/02/2016; Revisión: 16/07/2016; Aceptación: 9/09/2016

Resumen: En este trabajo se da a conocer una nueva ara con dedicación a la diosa Ataecina. La pieza, localizada hoy en Ońa (Burgos), probablemente proceda de la zona occidental de la provincia de Lusitania donde se ha recuperado la gran mayoría de las dedicaciones a esta deidad. El nombre de la diosa aparece acompańado, en este caso, por los epítetos Turebriga Proserpina, así como por el nombre del dedicante y, posiblemente, una fórmula votiva. Por su tipología y también por el material usado como soporte, su origen parece poder radicarse en el entorno de Mérida; sin embargo, debemos reconocer que la cronología y las circunstancias de su traslado nos son desconocidas. Creemos que el pequeño tamaño y el reducido peso del ara pudieron posibilitar un desplazamiento en la misma etapa romana, quizás como parte integrante del equipaje de un inmigrante; también es posible que su traslado se hubiera producido ya en época moderna por parte de un anticuario o bien de un particular.

Palabras clave: cultos locales; hábito epigráfico; epigrafía romana religiosa; Augusta Emerita; movilidad geográfica.

АвsтRACT: This paper presents a new altar dedicated to Ataecina. The object, located actually in Ońa (Burgos), it was probably moved from the West part of Lusitania where most of the dedications to this deity have been found. The name of the goddess, in this case, is followed by the epithets Turebriga Proserpina as well as the name of the devotee and possibly the votive formula. According to the material used as support and the typology of the altar its origin seems could have been manufactured in the area of Mérida, but circumstances and chronology of the displacement are unknown. We think that due to small size and reduced weight of the altar, it could have arrived during the Roman period as part of the luggage of an immigrant; but it could also have been moved in modern times by an antiques dealer or a particular.

Key words: local cults; epigraphic habit; Roman religious epigraphy; Augusta Emerita; geographic mobility.

\section{Introducción}

El árula que presentamos a continuación se conserva en el monasterio de San Salvador de Ońa (Burgos) $)^{1}$, en la sala capitular junto con otras

1 Este trabajo se ha realizado como parte del proyecto de tesis doctoral en el marco del Programa de Formación de Personal Investigador No Doctor del Gobierno Vasco, el programa de Contratación de Doctores Recientes del Vicerrectorado de Investigación de la UPV-EHU, el proyecto inscripciones también de época romana ${ }^{2}$. Desconocemos cuál fue su ubicación previa o cuándo fue

HAR2015-65526-P (MINECO/FEDER) y el Grupo de Investigación IT698-13.

2 Agradecemos al párroco del monasterio de Ońa, C. A. Haro Guerrero, y a A. J. Haro Hernández las facilidades prestadas para el acceso a las inscripciones y sus amables indicaciones; también damos las gracias a la Dra. M. C. González Rodríguez y a P. Fernández Madrid por habernos acompañado y ayudado en la autopsia de las piezas. Además, 
depositada allí, aunque el hecho de que no haya sido dada a conocer junto con el resto de inscripciones que ocupan la misma sala, publicadas durante los años $70^{3}$, y que tampoco haya sido documentada por E. Ortiz de Urbina en $1985^{4}$ nos hace pensar que, muy probablemente, haya llegado aquí en una fecha posterior ${ }^{5}$.

\section{Descripción}

Se trata de una pequeña árula (Fig. 1), cuyas dimensiones son $19 \times 10 \times 8,5 \mathrm{~cm}$, que presenta un buen estado de conservación general, aunque aparece desgastada por la erosión y con algunos golpes en sus aristas laterales, en el coronamiento y la base ${ }^{6}$. Estas zonas fracturadas permiten apreciar mejor el material en el que se ha realizado, mármol de color blanco sin vetas aparentes y grano grueso que, en la mayor parte de la pieza, presenta una pátina de tono ocre. Aunque algo deterioradas, todas las caras presentan molduras tanto en la parte superior como en la inferior. La base - $5 \mathrm{~cm}-$, en la actualidad desgastada y fracturada en la cara trasera, parece que pudo haber sido recta y se une a la parte central por medio de molduras de pequeño tamaño -escocia/ toro-. La parte central mide $8 \times 10,5 \mathrm{~cm}$ y tiene

expresamos nuestro agradecimiento a J. M. Abascal Palazón por la confirmación de que la dedicación correspondía a la diosa Ataecina y a los evaluadores y al comité editorial todas sus acertadas correcciones e indicaciones que han ayudado a mejorar la calidad del trabajo.

3 Las conocidas dedicaciones al dios Vurouius recogidas por el antiguo párroco de Oña, A. Lázaro, en los años 70 del siglo pasado en el entorno de Barcina de los Montes (AE, 1976 n. ${ }^{\text {os } 291, ~} 293$ y 294).

4 En ese momento, sin embargo, sí se documentaron dos inscripciones inéditas que fueron recogidas en su Memoria de Licenciatura presentada en 1985 pero sin publicar hasta fecha reciente (Fernández Corral, 2015).

5 Las conversaciones mantenidas con los distintos párrocos de la iglesia de San Salvador de Oña tampoco han permitido esclarecer este punto.

6 Con el objetivo de mostrar el volumen y las características del ara hemos realizado un modelo 3D que se encuentra disponible en https://sketchfab.com/mfernandezcorral.

(C) Universidad de Salamanca

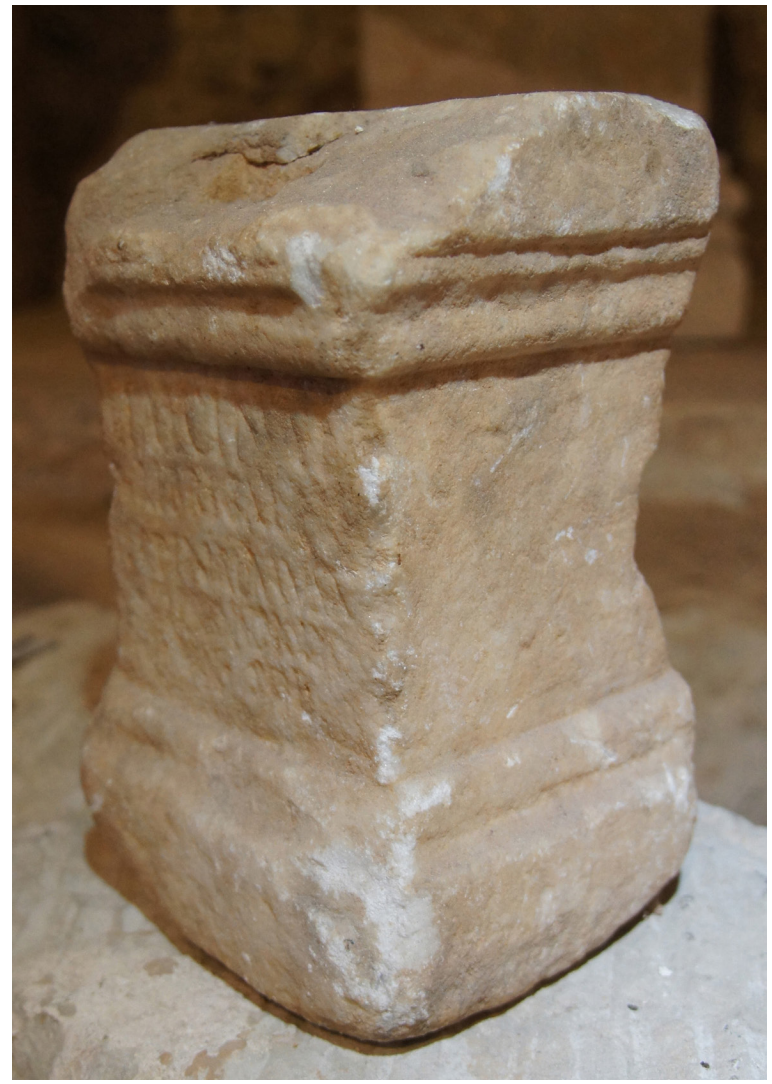

Fig. 1. Visión lateral del árula que permite observar las molduras.

algunos golpes en la parte trasera. El coronamiento $-6 \mathrm{~cm}$ - está formado por toro/escocia/toro y rematado con una moldura recta que se encuentra fracturada en la cara frontal del árula.

En la cara superior del ara encontramos dos oquedades rectangulares (Fig. 2) que no parecen corresponder a un focus fracturado, sino que podrían relacionarse con otras similares presentes en algunas aras de Santa Lucía del Trampal (Alcuéscar, Cáceres). La identificación de estas muescas junto con la presencia de apliques inferiores en los exvotos dedicados a esta misma diosa ha hecho pensar que su función podría haber sido permitir la colocación de estos objetos sobre el ara $^{7}$.

El campo epigráfico $-5 \times 9 \mathrm{~cm}$ - se encuentra en la parte superior del neto del árula y está dividido en 5 líneas que mantienen un interlineado que oscila

Esta es la propuesta de Abascal, 1995: 95. 


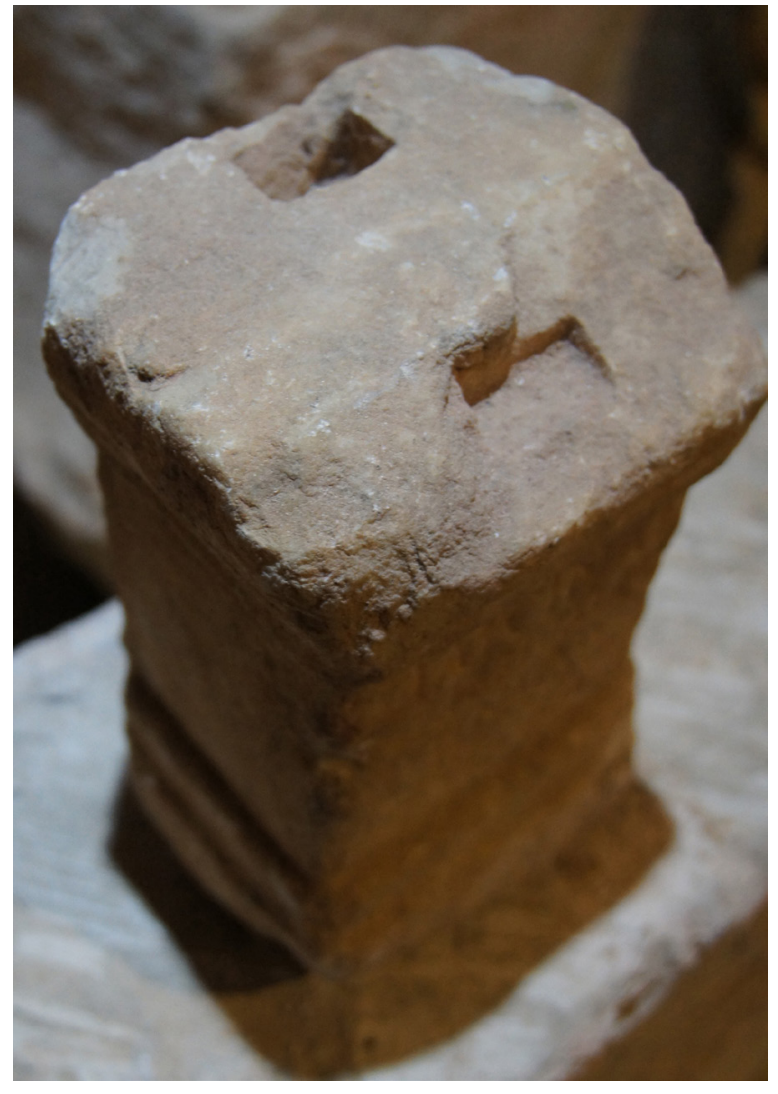

Fig. 2. Perspectiva del árula que permite observar las oquedades de su parte superior.

entre 0,2 y $0,5 \mathrm{~cm}$. Se encuentra muy erosionado y la lectura solo es posible por medio de la aplicación de luz rasante. Algunas letras del inicio de las líneas se han perdido o se encuentran muy desgastadas. Aunque es reconocible la existencia de una 4. ${ }^{a}$ línea con algunos trazos, su estado de erosión no permitido determinar a qué letras corresponde.

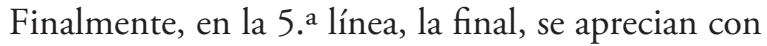
cierta claridad las letras sol que, por su posición en el texto y teniendo en cuenta el estado de la pieza, es probable que estuvieran precedidas por otras que actualmente no pueden ser identificadas.

Las letras son de pequeño tamańo y, por lo tanto, el surco de incisión es muy estrecho. El trazo es algo estilizado, un tanto irregular y no se observan nexos ni interpunciones. El tamaño de las letras es descendente, la $1 .^{\mathrm{a}}$ línea mide $1,5 \mathrm{~cm}$, mientras que las líneas $2 .^{\mathrm{a}}$ y $3 \mathrm{a}^{\mathrm{a}}$ alcanzan $1 \mathrm{~cm}$ y las dos últimas solo $0,7 \mathrm{~cm}$.

\section{Lectura e interpretación}

\author{
Ataecinae \\ [T]urebrigae Pro- \\ serpinae Musa \\ [---] \\ 5 [--- $] \operatorname{sol}($ uit)
}

Para Ataecina Turebriga Proserpina. Musa... cumplió (su voto?).

En las primeras líneas (Fig. 3) aparece el nombre de la divinidad indicado con su variante sorda Ataecina $^{8}$ y acompañada por el apelativo Turebriga seguido del teónimo latino Proserpina, cuyo culto parece haber estado asociado a esta deidad ${ }^{9}$. El estudio del resto de la estela descarta la presencia de otros apelativos propios de esta diosa como pueden ser Dea, Domina o Sancta.

Destaca el uso de la forma Turebrigae, desarrollado de forma completa, en lugar del acostumbrado Turibrigensis. Sin embargo, al observar el resto de dedicaciones en las que aparece este mismo epíteto, se observa que en la mayoría de las ocasiones no aparece escrito completamente, sino que ha sido desarrollado desde abreviaturas del tipo $T$ (uribrigensis) ${ }^{10}$, Tu(ribrigensis) $)^{11}$, Tur(ibrigensis) ${ }^{12}$, Turib(rigensis $)^{13}$, Turibr(igensis) $)^{14}$, Turibri(gensis) $)^{15}$, Turibrig(ensis) ${ }^{16}$

8 Sobre las diferentes variantes del teónimo $c f$. Abascal, 1995: 92-93 y 2002: 54-55.

9 También atestiguado en CIL II $462=$ ILS 451=AE 1959, 30; ERBeturi $89=$ HEP 6, $142=$ AE 1997, 815; CIL II 461 y $\operatorname{HE} \$ 5$, 76. Sobre su identificación con esta diosa latina cf. Salinas y Rodríguez Cortés, 2004: 286-291. No es nuestro objetivo hacer un análisis exhaustivo del culto a esta divinidad, sino señalar aquellas cuestiones aportadas por este nuevo hallazgo. Sobre su culto y la información disponible al respecto $c f$. principalmente los trabajos de Abascal, 1995, 2002, 2011 y de García-Bellido, 2001.

10 CIL X 7557; CIL II 461; CIL II 5299; ILER 744; CPILCáceres 139 y 332; CIL II 5298, AE 1946, 193, AE 1950, 14, ILER 743, CPILCáceres 138 y 331; Abascal, 1995, n. ${ }^{\circ} 5$.

${ }^{11}$ HEP 6, $142=A E$ 1997, 815 .

12 CIL II 5877; ILER 738 y 1008; Abascal, 1995, n. 7.

13 EE 9, 43, ILER 733.

14 CPILCáceres 432 y Abascal, 1995, n.o 8.

15 Abascal, 1995, n. ${ }^{\text {ss } 3, ~ 4, ~ 9, ~} 10$ y 14.

16 CIL II 462 = ILS 4515, AE 1959, 30; CIL II 605 = add. ILS 4515, EE 9, 42, ILER 743 . 


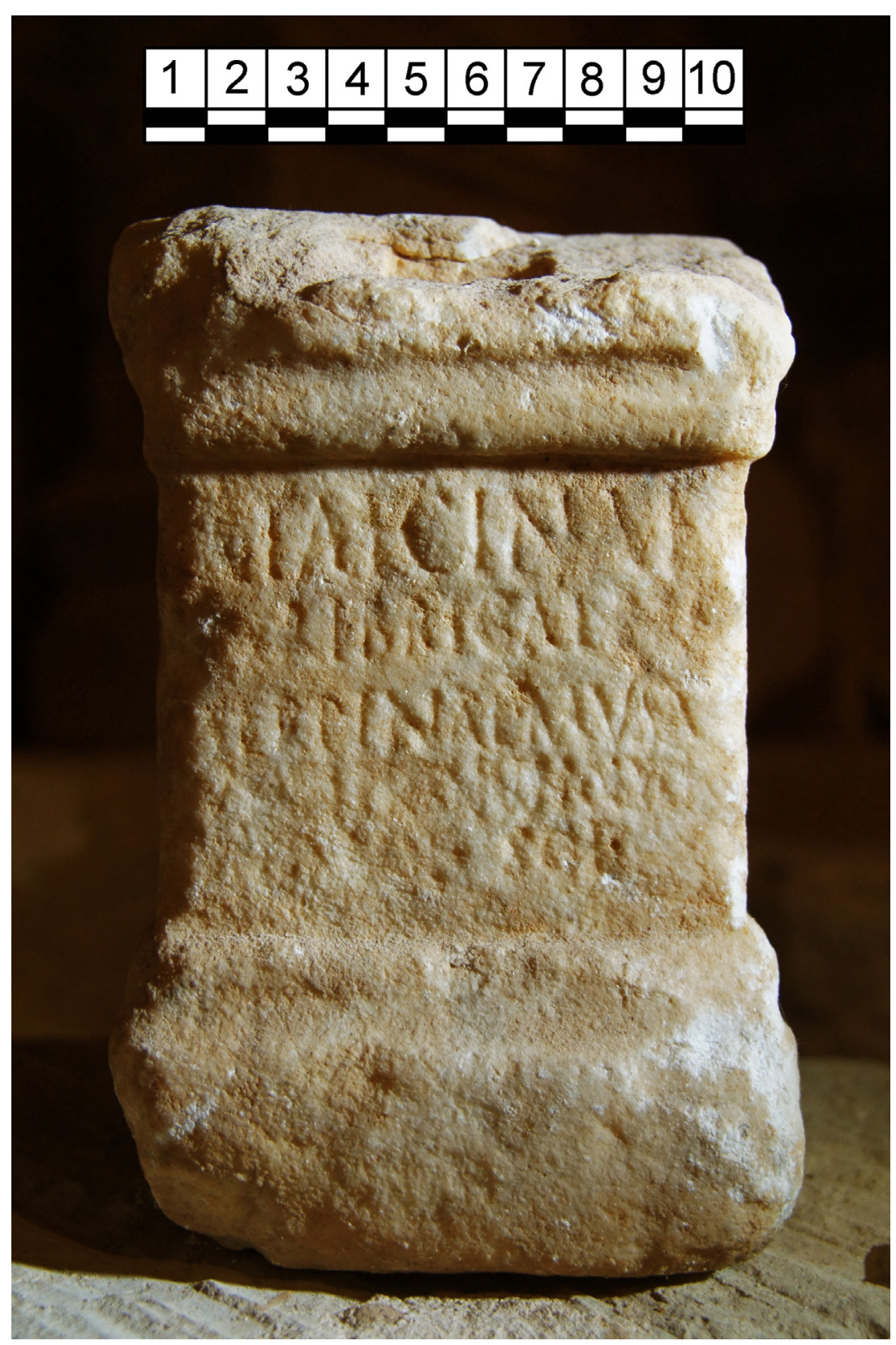

Fig. 3. Vista frontal del árula con la dedicación a Ataecina.

o Turibriclge(nsis $)^{17}$. Es por ello que consideramos que su desarrollo como Turibrigensis se trata, por lo general, de una convención epigráfica justificada por el carácter del término. Ahora bien, aunque el significado del epíteto en su forma de gentilicio o de

\footnotetext{
17 CIL II 71, ILER 740, IRCP 287; ILER 737; CPILCáceres
} 263.

(C) Universidad de Salamanca adjetivo no cambia su sentido, este nuevo hallazgo en su forma Turebrigae-que se añade a los otros conocidos previamente- ${ }^{18}$ debe hacernos pensar que su uso también estuvo extendido.

La 3. ${ }^{a}$ línea ofrece el nombre del dedicante, Musa, un cognomen femenino con varios testimonios más en la provincia de Lusitania. No obstante, dado el mal estado de conservación de la 4. ${ }^{a}$ línea del texto, no podemos descartar que el nombre continuara y se tratase de un hombre, quizás Musaeus ${ }^{19}$. En ambos casos estaríamos ante un cognomen griego que podría estar indicando una condición u origen servil del dedicante ${ }^{20}$.

Respecto a las dos últimas líneas, su lectura es complicada debido a su pequeño tamaño y el desgaste que han sufrido. Hemos descartado cualquier interpretación de la 4. ${ }^{\mathrm{a}}$ línea debido a que los trazos se confunden con una u otra letra dependiendo del punto desde el que se mire y se le enfoque la luz.

Respecto a la última línea, la presencia de las letras sol en el contexto de un ara dedicada a una deidad hace que sea muy probable que se trate del verbo soluere, propio de este tipo de monumentos que, además, también aparece en última posición en otras aras más dedicadas a esta misma diosa formando parte de fórmulas como animo libens uotum soluit y similares ${ }^{21}$.

\footnotetext{
18 EE 9, 42 y HEp 1, 81.

19 Abascal, 1994: 434; Navarro y Ramírez, 2003: 244.

20 Solin, 2003: 417-421.

${ }^{21}$ Abascal, 1995, n. ${ }^{\text {os }} 1,4,6,9,20,21$ y HEp 5, 1995, 76.
} 


\section{Localización y procedencia de la pieza}

Una de las principales cuestiones que destacan de este hallazgo es su lugar de conservación. Como bien es sabido, la mayoría de las aras dedicadas a Ataecina han sido localizadas en la provincia lusitana, concentrándose especialmente en Santa Lucía del Trampal (Alcuéscar, Cáceres) ${ }^{22}$ y sus inmediaciones, que incluyen la ciudad de Augusta Emerita ${ }^{23}$. Debido a la distribución de sus hallazgos (Fig. 4) se ha considerado un culto local, posiblemente ligado al desconocido núcleo de Turobriga al que parece referirse el epíteto de la deidad ${ }^{24}$, que habría alcanzado cierta difusión en la región como consecuencia de la fundación de Augusta Emerita y el desarrollo de la red viaria lusitana ${ }^{25}$.

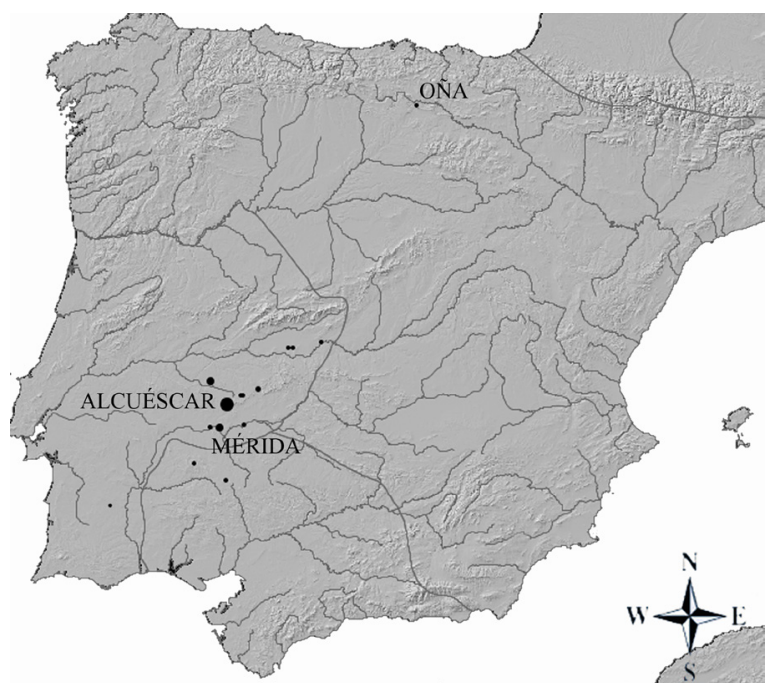

Fig. 4. Distribución de epigrafes con dedicaciones a Ataecina.

22 Abascal, 1995

23 Se considera que podría haber hasta tres centros de culto: uno en Santa María del Trampal, otro en la Dehesa Zafrilla de Malpartida (Cáceres) y el tercero en la Dehesa El Palacio de Herguijuela (Cáceres); cf. Abascal, 2002: 55-56.

24 Núcleo mencionado por Plinio $(\mathrm{NH} 3,14)$ entre las ciudades de la Bética céltica. Sobre la discusión en torno a su ubicación $c f$. Abascal, 1995: 97-101 y 2002: 57.

25 Tal y como proponen Salinas y Rodríguez Cortés, 2004: 286-291. Olivares, 2003: 306-310 vincula su difusión a la colonia y a la redistribución de población que habría supuesto su fundación.
Dadas las características del ara y su material, desconocido entre los monumentos epigráficos de la región, creemos conveniente descartar la posibilidad de que se tratase de un ara realizada en el entorno de Oña por un inmigrante. En su lugar, y dado el pequeño tamańo y peso de la pieza, creemos que es probable que fuera transportada desde su ámbito de culto conocido en un momento cronológico que, por el momento, no resulta posible determinar.

Su material y tipología no se asemejan al conjunto de Santa Lucía del Trampal, formado por aras de granito con molduras poco marcadas ${ }^{26}$, sino que recuerdan más a las dedicaciones localizadas en Mérida y su entorno. Los modelos más cercanos a la pieza son dos aras recuperadas en la colonia $(E E I X 42 \text { y } 43)^{27}$, un ara fracturada localizada en La Garrovilla (Badajoz) (HEP $5,1995,76)$ y dos aras más de la capital provincial que, aunque existen dudas sobre si hacen referencia a Ataecina $^{28}$, son también similares en material y tipología ( $A E$ 1983, 486 y HEp 2, 1990, 34) ${ }^{29}$.

Su mención junto a Proserpina también podría estar señalándonos su origen emeritense ya que se considera que ambas divinidades habrían sido asociadas en esta ciudad. Si bien es cierto que no parece que llegase a darse una total identificación de una y otra, la distribución de los epígrafes con dedicaciones religiosas a estas divinidades indica que la capital provincial lusitana pudo ser el lugar en el que ambos cultos estuvieron en contacto ${ }^{30}$.

Creemos difícil establecer la cronología en la que se trasladó el ara y cuáles fueron sus circunstancias.

26 Abascal, 1995: 67-75.

27 Puede verse un modelo 3D en www.epigraphia3d. es/3d-55.html.

28 Ambas fueron descartadas por Abascal (1995: 82) como dedicaciones seguras a Ataecina dada la imprecisión de la invocación.

29 Un modelo 3D de las mismas está disponible en www.epigraphia3d.es/3d-04.html y www.epigraphia3d.es/ 3d-57.html. Nos gustaría agradecer al investigador responsable del proyecto, M. Ramírez Sánchez, habernos facilitado las fotografías originales de los modelos 3D que nos han permitido realizar un estudio más detenido de las piezas, así como habernos alentado a realizar nuestro propio modelo 3D. Sobre el método empleado $c f$. Ramírez et al., 2014.

30 Abascal, 1995: 97. 
Este trasvase pudo haberse producido en época romana en el contexto de un viaje o cambio de residencia de un devoto procedente de su área de culto lusitana que podría haberlo llevado como parte de su equipaje. No obstante, también pudo ocurrir en época moderna, posiblemente por parte de un anticuario o incluso un particular, por ejemplo, en el contexto de un desplazamiento desde las zonas rurales a las áreas industriales del $\mathrm{N}$ peninsular.

La movilidad geográfica de época romana es un fenómeno demográfico sobradamente constatado, especialmente, a partir de las fuentes epigráficas ${ }^{31}$. $\mathrm{Si}$ bien es cierto que en contextos migratorios se han documentado testimonios en los que los inmigrantes realizaban dedicaciones, por lo general, a los dioses locales del lugar al que se desplazaban, también contamos con otros casos en los que se han trasladado aras realizadas previamente como parte del equipaje de un individuo, en especial, en el caso de militares $^{32}$. Dado el pequeño tamaño y peso del ara que presentamos, no descartamos que esta pudiera haber sido parte de un santuario doméstico de carácter privado y que, en un momento dado, hubiera sido trasladada junto con su devoto ${ }^{33}$.

Sin embargo, a pesar de que, como acabamos de decir, este tipo de traslados de aras de pequeño tamaño están atestiguados en época romana, también lo están los desplazamientos de epígrafes antiguos en época moderna. A modo de ejemplo, citaremos otra dedicación a Ataecina que formaba parte del conjunto epigráfico del yacimiento romano de Segobriga (Cuenca) (CIL II, 5877). Los datos disponibles situaban su descubrimiento en la muralla de Uclés

31 Este tema ha sido tratado, entre otros, por Iglesias y Ruiz Gutiérrez (2011) y en el dosier monográfico n. ${ }^{\circ} 30$ de Veleia. Su alcance y análisis es además el objetivo del proyecto de investigación MIGRA. Movilidad, arraigo y registro epigráfico de la memoria en la Hispania romana: estudio histórico y cartográfico dirigido por A. Ruiz Gutiérrez de la Univ. de Cantabria.

32 Olivares, 2003: 303-305. Como tal vez pudo ocurrir con la dedicación a Ataecina localizada en Cagliari (Cerdeña) (CIL X 7557) que, según Abascal (2002: n. 16), podría estar relacionada con la presencia de una unidad de auxilia Hispanorum.

33 Olivares, 2013.
(Cuenca), pero el hecho de ser la única pieza en granito de todo el conjunto y a su lejanía respecto el área de culto de esta diosa hizo que surgieran serias dudas sobre su verdadera procedencia ${ }^{34}$. Estas sospechas han podido confirmarse recientemente gracias a la localización de un dibujo anónimo de un manuscrito del s. xix depositado en el Archivo de la Real Academia de la Historia (RAH-9-7363-78) en el que se señala una localización acorde con sus características: Alconétar (Cáceres) ${ }^{35}$.

Es por ello que, aunque creemos muy probable que el ara fuera transportada desde el área de Mérida, ante la ausencia de datos esclarecedores, no podemos aventurarnos a proponer una cronología y circunstancias de desplazamiento concretas.

\section{Conclusiones}

El análisis directo de esta ara ha permitido reconocer una nueva dedicación a Ataecina lejos de su lugar de culto habitual, concentrado al o de la provincia de Lusitania. Como hemos señalado, no creemos que debamos pensar en un testimonio de su culto en época antigua fuera de su lugar habitual, sino que, dado el pequeño tamaño de la pieza, podría haber sido trasladada hasta Oña en un momento y circunstancia que no podemos determinar.

La diosa aparece mencionada con epítetos ya documentados en otras de sus dedicaciones como son Turebriga y Proserpina, ambos escritos de forma completa y, por lo tanto, sin dudas sobre su interpretación. El primero de ellos, además, invita a pensar que la habitual interpretación de sus diferentes abreviaturas podría no ser siempre Turibrigensis, sino que la forma que aparece aquí podría estar también extendida.

El nombre del dedicante podría ser un antropónimo griego como Musa, aunque el mal estado de conservación de la $4 .^{a}$ línea podría esconder un nombre más largo como Musaeus. Finalmente, aunque incompleta, la presencia de la fórmula soluit en la última línea indica la presencia de una fórmula votiva final.

34 Almagro Gorbea, 1995: n. 3.

35 Abascal y Cebrián, 2004-2005: 202-203. 


\author{
Abreviaturas \\ AE = L'Année Épigraphique. \\ $C I L=$ Corpus Inscriptionum Latinarum. \\ CPILCáceres = HuRTAdo de SAN ANTONIO, R. (1977): \\ Corpus Provincial de Inscripciones Latinas (Cáceres). \\ Cáceres. \\ $E E=$ Ephemeris Epigraphica . \\ ERBetur = CANTO, A. M. (1997): Epigrafía Romana de \\ la Beturia céltica. Madrid. \\ HEp = Hispania Epigraphica. \\ ILER = VIVES, J. (1971-72): Inscripciones latinas de la \\ España romana. Barcelona. \\ ILS = Dessau, H. (1892-1916): Inscriptiones Latinae \\ Selectae. Berlin. \\ $I R C P=$ ENCARNAÇÃo, J. (1984): Inscriçóes romanas do \\ Conventus Pacensis: subsidios para o estudo da ro- \\ manizaçâo. Coimbra.
}

\section{Bibliografía}

Abascal, J. M. (1994): Los nombres personales en las inscripciones latinas de Hispania. Murcia: Univ. de Murcia.

Abascal, J. M. (1995): "Las inscripciones latinas de Santa Lucía del Trampal (Alcuéscar, Cáceres) y el culto de Ataecina en Hispania", Archivo Español de Arqueología, 68, pp. 31-106.

Abascal, J. M. (2002): “Ataecina”. En Raposo, L. (ed.): Religióes da Lusitania. Loquuntur saxa. Catálogo de la exposición. Lisboa: Museu Nacional de Arqueologia: Ministério da Cultura, Instituto Português de Museus, pp. 53-60.

Abascal, J. M. (2011): 'La Dea Domina Sancta Turibrigensis Ataecina y las nuevas evidencias epigráficas de Alcuéscar (Cáceres)". En CARdim, J. (coord.): Diis deabusque. Actas II Colóquio internacional de epigrafia 'Culto y Sociedade' (Sintra, 1995). São Miguel de Odrinhas: Museo Arqueológico, pp. 15-36.

Abascal, J. M. y Cebrián, R. (2004-2005): "Inscripciones de Guadalajara, Ávila, Alconetar, Segobriga y
Sevilla en manuscritos de la Real Academia de la Historia", Lucentum, 23-24, pp. 197-205.

Almagro Gorbea, M. (1995): "El Lucus Dianae con inscripciones rupestres de Segobriga". En RodRígueZ Colmenero, A. y Gasperini, L. (eds.): Saxa scripta (inscripciones en roca). Actas Simposio Internacional Ibero-Itálico sobre epigrafía rupestre. A Coruña: Ediciós do Castro, pp. 61-98.

Fernández Corral, M. (2015): "Dos inscripciones funerarias y una estela oikomorfa en Ońa (Burgos)", Ficheiro Epigrafico, 132, pp. 552-554.

García-Bellido, M. P. (2001): "Lucus Feroniae Emeritensis”, Archivo Español de Arqueología, 74, pp. 53-71.

Iglesias, J. M. y Ruiz Gutiérrez, A. (2011): Viajes y cambios de residencia en el mundo romano. Santander: publican.

Navarro, M. y Ramírez, J. L. (coords.) (2003): Atlas antroponímico de la Lusitania romana. MéridaBordeaux: Fundación de Estudios RomanosAusonius Éditions.

Olivares, J. C. (2003): "Reflexiones sobre las ofrendas votivas a dioses indígenas en Hispania: ámbitos de culto y movimientos de población", Veleia, 20, pp. 297-313.

Olivares, J. C. (2013): "La omisión del dedicante en las inscripciones votivas de Hispania como indicio de su ubicación en ámbitos privados", Studia Historica. Historia Antigua, 31, pp. 59-87.

Salinas, M. y Rodríguez Cortés, J. (2004): “Corrientes religiosas y medios de comunicación en Lusitania durante el Imperio romano". En Gorges, J.-G.; Cerrillo, E. y Nogales, T. (coords.): Actas V Mesa redonda internacional sobre Lusitania romana: las comunicaciones (Cáceres, 2002). Madrid: Ministerio de Cultura, pp. 277-291.

Ramírez, M.; SuÁrez, J. P. y Castellano M. A. (2014): "Epigrafía digital: tecnología 3D de bajo coste para la digitalización de inscripciones y su acceso desde ordenadores y dispositivos móviles", El profesional de la Información, 23/5, pp. 467-474.

Solin, H. (2003): Die griechischen Personennamen in Rom. Ein Namenbuch, Zweite, völlig neu bearbeitete Auflage. Berlin-New York: Walter de Gruyter. 